$$
\text { "tmcs-tuzson" — 2013/10/21 — 15:13 — page } 231 \text { — \#1 }
$$

\title{
The study of sequences defined by a first order recursion by means of a pocket calculator
}

\author{
ZOLTÁN TUZSON
}

\begin{abstract}
This paper will present the way we can use a simple pocket calculator to teach mathematics. Namely, a pocket calculator can be very useful to study the properties of sequences defined by first order recursion (e.g. monotonicity, boundedness and convergence) and to gain a deeper understanding.
\end{abstract}

Key words and phrases: sequence, bounded, monotonicity, limit, pocket calculator, convergence.

ZDM Subject Classification: I30, N20, D10.

Nowadays, one can propose whether calculators (i.e., that of mobile phones or computers) can be used for any other purposes than ordinary arithmetic? This paper will prove that it can be very beneficial for studying the convergence of sequences as well as for guessing the limit of a sequence. Moreover in the second part it plays an important role, since it can create predictions regarding the limit of a sequence.

Therefore, my main aim is to advertise and to recommend trying it out.

The purpose of my experiments was to study the convergence of certain sequences defined by a recursive relation with the students. They have previously gained the more important, necessary concepts and knowledge, the information related to monotonicity and boundedness, and we had started to calculate the limits of sequences. But we can say that we are only beginners in this domain.

Copyright (C) 2013 by University of Debrecen 
In my opinion the whole mathematical analysis of High School, especially the convergence of sequences and the calculation of their limits is hard to understand because a proper concrete illustrative model is missing. More exactly, the phenomena related to infinite cannot really be modelled. We do not have any intuitive basis or support for this, only definitions, concepts, predefined rules and theorems. That's why I wondered how we can present this topic in such a way that the students can experience it actively. Then it came to my mind that with a manual calculator we can experience such concepts as monotonicity, boundedness and, finally, the concept of convergence quickly and easily. For this, I decided that I will try all this, which I am going to share below. I must admit that I did not regret it because according to the feedback, the students understood this topic better than if I had just written the lesson on the blackboard with a chalk. At the same time, we also presented and analyzed in detail the following proof on the blackboard. At first, however, we tried the experiment and after that we discussed and proved our results.

In what follows, we will use the so called Weierstrass theorem which states that each monotone and bounded sequence of a real number $\left(x_{n}\right)_{n \geq 1}$ is convergent. The key to the proof of the theorem is that in the case of each bounded and decreasing $\left(x_{n}\right)_{n \geq 1}$ sequence of a real number $\lim _{n \rightarrow \infty} x_{n}=\inf \left\{x_{n}: n \geq 1\right\}$, or in the case of each bounded and increasing $\left(x_{n}\right)_{n \geq 1}$ sequence of real numbers $\lim _{n \rightarrow \infty} x_{n}=\sup \left\{x_{n}: n \geq 1\right\}$ (see e.g. [1]).

EXPERIMENT 1. Let's type 2 into a pocket calculator and calculate its square root. Then calculate the square root of the result. We do this until we see the same number displayed over and over again. What can we say about the monotonicity, boundedness and limit of the sequence? How can we explain what we can see?

Solution. On the display of a pocket calculator which can display only 8 characters we can see the following, respectively: $1.4141356 ; 1.1892071 ; 1.0905076$; $1.0442737 ; 1.0218971 ; 1.0108892 ; 1.0054298 ; 1.0027112 ; 1.0013546 ; 1.0006770$; $1.0003384 ; 1.0001691 ; 1.0000845 ; 1.0000422 ; 1.0000210 ; 1.0000104 ; 1.0000051$; $1.0000025 ; 1.0000012 ; 1.0000005 ; 1.0000002 ; 1.0000001 ; 1 . ; 1 . ; 1 . ; \ldots$.

The following properties can be read:

(1) The resulting sequence is strictly decreasing. We are going to prove this. The members of the sequence in question are: $a_{1}=\sqrt{2}=2^{\frac{1}{2}}, a_{2}=\sqrt{a_{1}}=\sqrt{\sqrt{2}}=$ 


$$
\begin{aligned}
& =2^{\frac{1}{2^{2}}}, a_{3}=\sqrt{a_{2}}=\sqrt{\sqrt{\sqrt{2}}}=2^{\frac{1}{2^{3}}} \text {, and usually }
\end{aligned}
$$

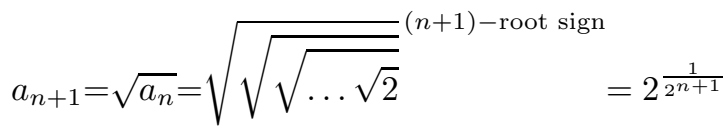

for $\forall n \geq 0$. It is easy to see that $a_{1}>\sqrt{a_{1}}=a_{2}$, hence if we use the method of mathematical induction and we assume that $a_{n+1}>a_{n}$, then the

$$
a_{n+1}-a_{n}=\sqrt{a_{n}}-\sqrt{a_{n-1}}=\frac{a_{n}-a_{n-1}}{\sqrt{a_{n}}+\sqrt{a_{n-1}}}
$$

formula immediately implies that $a_{n}>a_{n+1}$, namely, the sequence is indeed strictly decreasing. Another way to prove the monotonicity of the sequence is if we write

$$
\frac{a_{n+1}}{a_{n}}=\frac{2^{\frac{1}{2^{n+1}}}}{2^{\frac{1}{2^{n}}}}=2^{-\frac{1}{2^{n+1}}}<2^{0}=1 .
$$

This is shorter than the previous proof, which we present in order to help to acquire the technique of proving.

(2) Because the sequence is strictly decreasing it has a least upper bound. In this way $a_{n} \leq a_{1}=\sqrt{2}, \forall n \geq 1$. And as each member of the sequence is positive we can surely conclude that $a_{n} \in(0, \sqrt{2}]$ for $\forall n \geq 1$. So the sequence is bounded.

(3) Since the sequence $\left(a_{n}\right)_{n \geq 1}$ is strictly decreasing and bounded, hence due to the theorem of Weierstrass, the sequence is convergent, so it has a limit. On the display of the pocket calculator we sense this, as after executing a certain number of operations the display shows 1 . From this, one might conjecture that $\lim _{n \rightarrow \infty} a_{n}=1$. Indeed, since for all $n \geq 1$ equation $a_{n+1}^{2}=a_{n}$ is fulfilled, so if $\lim _{n \rightarrow \infty} a_{n}=a$, then necessarily $a^{2}=a$ has to be satisfied. Since, however, $a_{n} \geq 1$ for all $n \geq 1$, we get that $a \geq 1$, so $a=1$. At the same time we have illustrated the theorem which says that the greatest lower bound of a monotonic decreasing sequence is its limit, in our case 1.

(4) Furthermore it has to be explained why, after executing a certain number of operations, there always appears 1 on the display of the calculator. We can find out the reason of this when we do these calculations on a calculator that has more than 8 digits on its display. Here, after 1,0000000 there can appear digits which are not a 0 . Therefore, with an 8 digit calculator we could only approximate the limit of the sequence which was 1 with seven decimals.

It is interesting to observe the members of the sequences $a_{1}=1+10^{-6}$, $a_{n+1}=a_{n}^{2}(n \geq 1)$ or $a_{1}=1-10^{-7}, a_{n+1}=a_{n}^{2}$ on the display of the calculator. 
EXPERIMENT 2. The task is the same as in Experiment 1, but with the difference that in the place of 2 we can pick an arbitrary number $a>0$.

Solution. Executing the calculations in specific cases and observing the shown values, we can see that after a number of steps the number 1 is shown again. The value 1 can be reached faster or slower, that is to say, even the "speed of convergence" changes. Every other result and the proof are the same as shown before. All in all, we showed that for arbitrary $a>0$

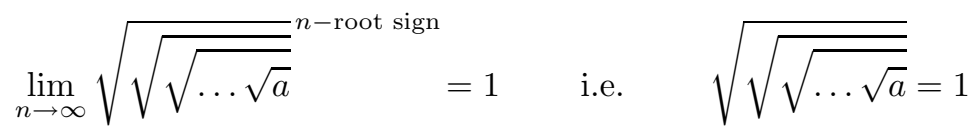

holds, where the number of the root signs is infinitely big.

EXPERIMENT 3. Let's type 2 into a pocket calculator and calculate its square root. Multiply the result by two and calculate its square root again. We shall repeat this operation until we get the same number displayed over and over again. What can we say about the monotonicity, boundedness and limit of the sequence? How can we explain what we can see?

Solution. On the display of a pocket calculator which can only display 8 characters we can see the following, respectively: $1.4141356 ; 1.6817928 ; 1.8340080$; 1,$9152065 ; 1.9571441 ; 1.9784560 ; 1.9891988 ; 1.9945921 ; 1.9972942 ; 1.9986466$; $1.9993232 ; 1.9996615 ; 1.9998307 ; 1.9999153 ; 1.9999576 ; 1.9999758 ; 1.9999884$; $1.9999947 ; 1.9999973 ; 1.9999986 ; 1.9999993 ; 1.9999996 ; 1.9999998 ; 1.9999999 ; 2$; $2 ; 2 ; \ldots$.

The following properties can be read:

(1) The resulting sequence is strictly increasing. We are going to prove this. The members of the sequence in question are: $a_{1}=\sqrt{2}=2^{\frac{1}{2}}, a_{2}=\sqrt{2 a_{1}}=$ $=\sqrt{2 \sqrt{2}}=2^{\frac{1}{2}+\frac{1}{4}}, a_{3}=\sqrt{2 a_{2}}=\sqrt{2 \sqrt{2 \sqrt{2}}}=2^{\frac{1}{2}+\frac{1}{4}+\frac{1}{8}}$, and usually

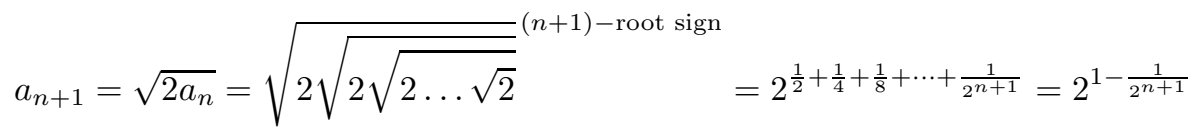

for $\forall n \geq 0$.

It is easy to see that on the basis of the relations $\sqrt{2}=a_{1}<\sqrt{2 a_{1}}=$ $=\sqrt{2 \sqrt{2}}=a_{2}$, and

$$
a_{n+1}-a_{n}=\sqrt{2 a_{n}}-\sqrt{2 a_{n-1}}=\frac{2\left(a_{n}-a_{n-1}\right)}{\sqrt{2 a_{n}}+\sqrt{2 a_{n-1}}}
$$




$$
\text { "tmcs-tuzson" — 2013/10/21 — 15:13 — page } 235 \text { — \#5 }
$$

The study of sequences defined by a first order recursion by means of a pocket calculator 235

we can prove by mathematical induction that the sequence is strictly increasing, indeed.

(2) Since the sequence is strictly increasing, it has a greatest lower bound, and thus $a_{n} \geq a_{1}=\sqrt{2}$ for $\forall n \geq 1$. On the basis of the display of the calculator we have a suspicion that the least upper bound is 2 , that is $a_{n}<2$ for $\forall n \geq 1$. This can be proved with the help of mathematical induction, as $a_{1}=\sqrt{2}<2$, and if we suppose that $a_{n}<2$, then $a_{n+1}=\sqrt{2 a_{n}}<\sqrt{2 \cdot 2}=2$. So $a_{n} \in[\sqrt{2}, 2)$ for $\forall n \geq 1$.

(3) Since the sequence $\left(a_{n}\right)_{n \geq 1}$ is strictly increasing and bounded, the theorem of Weierstrass yields that the sequence is convergent, that is, it has a limit. This can be seen on the calculator getting the number 2 on its display after a number of operations have been done. From this the equation $\lim _{n \rightarrow \infty} a_{n}=2$ can be concluded. Indeed, we know that for all $n \geq 1, a_{n+1}^{2}=2 a_{n}$ is valid. Thus, if $\lim _{n \rightarrow \infty} a_{n}=a$, then $a$ satisfies the algebraic equation for $a^{2}=2 a$. But $a_{n} \geq 1,(n \geq 1)$, that is why $a \geq 1$ and in this way $a=2$. At the same time another theorem is illustrated, according to which the least upper bound of an increasing sequence is the limit of the sequence, in our case this is 2 .

EXPERIMENT 4. The problem is the same as in the first two experiments, with the exception that we take a number $a>0$ instead of the value 2 .

Solution. Executing the calculations in specific cases and observing the shown values, we can see that after a number of steps the number $a$ is shown again. Every other result and the proof is the same as it was shown before, only $\lim _{n \rightarrow \infty} a_{n}=a$ changes according to $a_{1}=\sqrt{a}$. Summing up we proved that for arbitrarily fixed $a>0$,

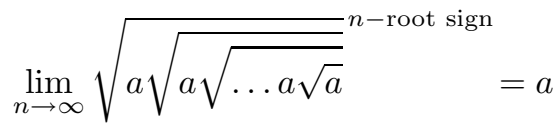

namely $\sqrt{a \sqrt{a \sqrt{\ldots a \sqrt{a}}}}=a$, where the number of the root signs is infinitely large.

EXPERIMENT 5. Let's type 2 into a pocket calculator and calculate its square root. Add 2 to the result and calculate its square root again. Repeat this operation until we get the same number reappearing on the display. What can we say about the monotonicity, boundedness and limit of the sequence? How can we explain what we can see? 
Solution. On the display of a pocket calculator which can only display 8 characters, in turns, we can see the following: $1.414135 ; 1.8477590 ; 1.9615705$; $1.9903694 ; 1.9975909 ; 1.9993976 ; 1.9998494 ; 1.9999623 ; 1.9999905 ; 1.9999976$; $1.9999994 ; 1.9999998 ; 1.9999999 ; 2 ; \mathbf{2} ; \mathbf{2} ; \ldots$

(1) The resulting sequence is strictly increasing, which we shall prove. The members of the sequence in question are: $a_{1}=\sqrt{2}, a_{2}=\sqrt{2+a_{1}}=\sqrt{2+\sqrt{2}}$, $a_{3}=\sqrt{2+a_{2}}=\sqrt{2+\sqrt{2+\sqrt{2}}}$, and in general

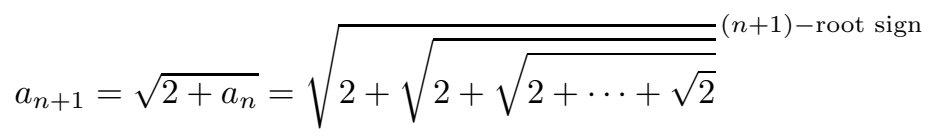

for $\forall n \geq 0$. We can see that in this case we get only a recursive relationship, we cannot write the general member algebraically, as in the previous cases. It is easy to see that on the basis of the relations $a_{1}=\sqrt{2}<\sqrt{2+\sqrt{2}}=a_{2}$, and

$$
a_{n+1}-a_{n}=\sqrt{2+a_{n}}-\sqrt{2+a_{n-1}}=\frac{a_{n}-a_{n-1}}{\sqrt{2+a_{n}}+\sqrt{2+a_{n-1}}}
$$

we can prove by mathematical induction that the sequence is strictly increasing.

(2) Since the sequence is strictly increasing it has a greatest lower bound, and thus $a_{n} \geq a_{1}=\sqrt{2}$ for $\forall n \geq 1$. On the basis of the calculator's display we believe that the least upper bound is 2 , namely $a_{n}<2$, for $\forall n \geq 1$. This can easily be proved by mathematical induction, because $a_{1}=\sqrt{2}<2$, and if we suppose that $a_{n}<2$, then $a_{n+1}=\sqrt{2+a_{n}}<\sqrt{2+2}=2$. So, $a_{n} \in[\sqrt{2}, 2)$ for $\forall n \geq 1$.

(3) Because the sequence $\left(a_{n}\right)_{n \geq 1}$ is strictly increasing and bounded, due to the Weierstrass theorem, it is also convergent, i.e. it has a limit. This can be seen on the calculator getting the number 2 on its display after a number of operations have been completed. This means that the $\lim _{n \rightarrow \infty} a_{n}=2$, which is true, because if $\lim _{n \rightarrow \infty} a_{n}=x$, then $\lim _{n \rightarrow \infty} a_{n+1}=x$ is also true. The limit satisfied $a_{n+1}=\sqrt{2+a_{n}}$ so $x=\sqrt{2+x} \Leftrightarrow x^{2}-x-2=0$ giving $x=2$, because $x=-1 \notin[\sqrt{2}, 2)$. Therefore $\lim _{n \rightarrow \infty} a_{n}=2$ which means, that

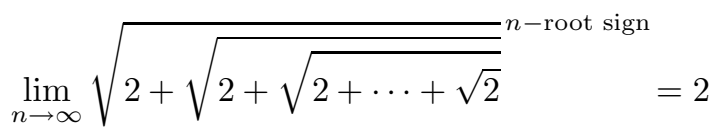


or otherwise

$$
\sqrt{2+\sqrt{2+\sqrt{2+\cdots+\sqrt{2+\ldots}}}}=2
$$

where the number of root-signs is infinitely large.

EXPERIMENT 6 . We take the number 6 instead of 2 in the previous exercise. What can be said about the monotonicity, boundedness and limit of the sequence? How can we explain what we can see?

Solution. The resulting sequence is $a_{1}=\sqrt{6}, a_{2}=\sqrt{6+a_{1}}=\sqrt{6+\sqrt{6}}$, $a_{3}=\sqrt{6+a_{2}}=\sqrt{6+\sqrt{6+\sqrt{6}}}$, and in general

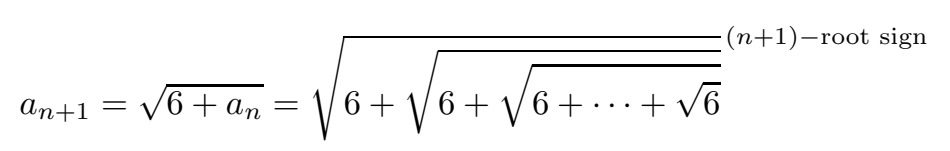

for $n \geq 0$. We are going to prove the same way as before that the sequence is strictly increasing. Therefore the greatest lower bound is the first member, namely $a_{n} \geq a_{1}=\sqrt{6}$, for $\forall n \geq 1$. This time we can see the least upper bound is 3 . We can easily prove this by mathematical induction, namely $a_{1}=\sqrt{6}<3$, and if we suppose, that $a_{n}<3$, then $a_{n+1}=\sqrt{6+a_{n}}<\sqrt{6+3}=3$. Therefore $a_{n} \in[\sqrt{6}, 3)$ for $\forall n \geq 1$. Since the $\left(a_{n}\right)_{n \geq 1}$ sequence is strictly increasing and bounded, on the basis of the Weierstrass theorem the sequence is convergent, that is, it has a limit. This can be seen on the display of the calculator showing the number 3 after a number of operations. This means that $\lim _{n \rightarrow \infty} a_{n}=3$, which on the basis of $a_{n+1}^{2}=6+a_{n}$ gives $x^{2}-x-6=0$ which has only one positive root, that being $x=3$.

REMARK 1. The reader is probably asking himself what kind of number we should choose besides 2 and 6 so that $\lim _{n \rightarrow \infty} a_{n}=k$, would be a natural number. The answer to this question is not that difficult, as the preceding sequences $a_{1}=$ $=\sqrt{a}, a_{n+1}^{2}=a+a_{n}, a>0$ were defined recursively, and if we consider the limit here, then we can see the result $a=k^{2}-k=(k-1) k$. Thus, if $a_{1}=\sqrt{a}$ and $a \in\{1 \cdot 2 ; 2 \cdot 3 ; 3 \cdot 4 ; \ldots ;(k-1) k ; \ldots\}_{k \in N^{*} \backslash\{1\}}$ then the limits of the sequence in question are going to be $2,3,4, \ldots, k, \ldots$ Experiments similar to the previous ones can be done.

EXPERIMENT 7. Let's type 1 into a pocket calculator and calculate its square root. Add 1 to the result and calculate its square root again. We shall repeat 
this operation until we get the same number reappearing on the display. What can we say about the monotonicity, boundedness and limit of the sequence? How can we explain what we can see?

Solution. On the display of a pocket calculator which can only display 8 characters, we can see the following: $1 ; 1.414135 ; 1.5537739,1.5980531 ; 1.6118477$; $1.6161212 ; 1.6174427 ; 1.6178512 ; 1.6179775 ; 1.6180165 ; 1.61802859 ; 1.6180323$; $1.6180334 ; 1.6180338 ; \mathbf{1 . 6 1 8 0 3 3 9} ; \mathbf{1 . 6 1 8 0 3 3 9} ; \mathbf{1 . 6 1 8 0 3 3 9} ; \ldots$ This time for a "constant" number we didn't get an integer value, but the number 1.6180339, which is of course only a seven decimal approximation.

Our sequence this time is $a_{1}=\sqrt{1}, a_{2}=\sqrt{1+a_{1}}=\sqrt{1+\sqrt{1}}=\sqrt{2}, a_{3}=$ $=\sqrt{1+a_{2}}=\sqrt{1+\sqrt{1+\sqrt{1}}}$, and usually

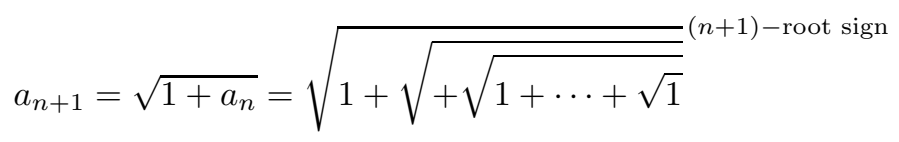

for $\forall n \geq 0$. According to the computed values of the sequence it is conjectured to be strictly increasing. This can be proved by mathematical induction similarly to the previous cases with the

$$
a_{n+1}-a_{n}=\sqrt{1+a_{n}}-\sqrt{1+a_{n-1}}=\frac{a_{n}-a_{n-1}}{\sqrt{1+a_{n}}+\sqrt{1+a_{n-1}}}
$$

relation. Since the sequence is strictly increasing, the greatest lower bound is the first member, namely $a_{n} \geq a_{1}=1$, for $\forall n \geq 1$. Our guess regarding the upper bound is that an approximate value of the upper bound is $1.6180339 \ldots$ If this proved to be correct, then the sequence would be monotonic and bounded, thus there would exist $\lim _{n \rightarrow \infty} a_{n}=x$, which on the basis of $a_{n+1}^{2}=1+a_{n}$ gives $x^{2}-x-1=0$, which has exactly one positive root of $x=\frac{1+\sqrt{5}}{2}=1.6180339 \ldots$ agreeing with the calculator. Thus, the least upper bound of the sequence is the number $\frac{1+\sqrt{5}}{2}=\mathbf{1 . 6 1 8 0 3 3 9} \ldots$ (this number is also called the golden ratio), and we can prove this by induction, if we assume, that $a_{n}<\frac{1+\sqrt{5}}{2}$, then $a_{n+1}=$ $=\sqrt{1+a_{n}}<\sqrt{1+\frac{1+\sqrt{5}}{2}}=\frac{1+\sqrt{5}}{2}$.

REMARK 2. The preceding sequences $a_{1}=\sqrt{a}, a_{n+1}=\sqrt{a+a_{n}}, a>0$ were defined by a recursive relation. It can be easily proved, that this sequence is strictly increasing and bounded. More precisely, for all $n \geq 1 a_{n} \in\left[\sqrt{a}, \frac{1+\sqrt{4 a+1}}{2}[\right.$. Therefore, the sequence is convergent as well, and if $\lim _{n \rightarrow \infty} a_{n}=x$, then this gives $x=\sqrt{a+x} \Leftrightarrow x^{2}-x-a=0$, which has a positive root $x=\frac{1+\sqrt{4 a+1}}{2}$. Based on 


$$
\text { "tmcs-tuzson" — 2013/10/21 — 15:13 — page } 239 \text { — \#9 }
$$

The study of sequences defined by a first order recursion by means of a pocket calculator 239

Remark 1, this limit will only be an integer number for $a \in N^{*}$ if $a=(k-1) k$ and $k \in N^{*} \backslash\{1\}$, in every other case it will be an irrational number (see for instance in $[1])$.

EXPERIMENT 8. Let's type the reciprocal of 1 into a pocket calculator and add 1 to it. To the reciprocal of the result let's add 1 again, and to the reciprocal of this result add 1 for a third time. Repeat this operation until the number on the display does not change. What can we say about the monotonicity, boundedness and limit of the sequence? How can we explain what we can see?

Solution. On the display of a pocket calculator which can display only 8 characters, in turns, we can see the following: $2 ; 1.5000000 ; 1.6666666 ; 1.6000000$; $1.6250000 ; 1.6153846 ; 1.6190476 ; 1.6176470 ; 1.6181818 ; 1.6179775 ; 1.6180555$; $1.6180257 ; 1.6180371 ; 1.6180328 ; 1.6180344 ; 1.6180338 ; 1.6180340 ; \mathbf{1 . 6 1 8 0 3 3 9}$; $1.6180339 ; 1.6180339 ; \ldots$

We defined the sequence as: $a_{1}=1, a_{2}=\frac{1}{a_{1}}+1, a_{3}=\frac{1}{a_{2}}+1$, and in general $a_{n+1}=\frac{1}{a_{n}}+1$, for $\forall n \geq 1$. If we follow the numbers on the display we can see, that the sequence is neither increasing nor decreasing, but $a_{1}<a_{3}<a_{5}<$ $\cdots<a_{2 k-1}<\ldots$ and $a_{2}>a_{4}>a_{6}>\ldots>a_{2 k}>\ldots$ i.e. the odd members of the sequence form a subsequence which is strictly increasing, and the other subsequence formed by the even members is strictly decreasing. We are going to prove this result. The recursion shows that

$$
a_{n+2}-a_{n}=\left(\frac{1}{a_{n+1}}+1\right)-\left(\frac{1}{a_{n-1}}+1\right)=\frac{1}{a_{n+1} a_{n-1} a_{n} a_{n-2}}\left(a_{n}-a_{n-2}\right),
$$

and we apply mathematical induction. Regarding boundedness, we can prove that $1<a_{2 k-1}<\frac{1+\sqrt{5}}{2}<a_{2 k}<2$. Therefore the sequence is bounded, thus due to the Weierstrass theorem, it is convergent, and so has a limit. Let there be $\lim _{n \rightarrow \infty} a_{n}=x$, so based on the $a_{n+1}=\frac{1}{a_{n}}+1$ recursion $x=\frac{1}{x}+1 \Leftrightarrow x^{2}-x-1=$ $=0$, which has only one positive root $x=\frac{1+\sqrt{5}}{2} \approx 1.6180339 \ldots$. On the display of the calculator, this value was got with a 7 decimal precision.

REMARK 3. As in the previous experiment, in the place of $a_{1} \neq 1$ we would put any arbitrary number. The sequence will still be defined by $a_{n+1}=\frac{1}{a_{n}}+a$ recursion, and according to what we have proved before, the same results can be derived with $\frac{1+\sqrt{5}}{2}$ replaced by $\frac{a+\sqrt{a^{2}+4}}{2}$.

REMARK 4. It is well known that we define the Fibonacci sequence as: $f_{1}=$ $=f_{2}=1$ and $f_{n+2}=f_{n+1}+f_{n}$ for $\forall n \geq 1$. We can produce a sequence of the 
ratios of two successive members from the Fibonacci sequence, namely $\frac{f_{k+1}}{f_{k}}=a_{k}$ for $\forall k \geq 1$. Then $f_{n+2}=f_{n+1}+1 \Leftrightarrow a_{n+1}=\frac{1}{a_{n}}+1$ that is to say, that we have generated the sequence from the previous experiment. Based on that experiment, the sequence produced of the ratios of two successive members from the Fibonacci sequence is convergent and its limit is $\frac{1+\sqrt{5}}{2}$.

Finally, we will notice that the convergence of numerous other sequences defined by first order recursion can be studied with a pocket calculator. For the interested reader we recommend a study of the convergence of the sequences defined by first order recursion in the cases offered below, using the model shown before:

1) $a_{1}=a \geq \frac{3}{4}$ and $a_{n+1}=\sqrt{4 a_{n}-3}$ for $\forall n \geq 1$.

2) $a_{1}=a>0, k>0, a_{n+1}=\sqrt{a_{n}}+k$ for $\forall n \geq 1$.

3) $a_{1}>0, k>0, a_{n+1}=\frac{1}{2}\left(a_{n}+\frac{k}{a_{n}}\right)$ for $\forall n \geq 1$.

\section{References}

[1] V. A. Ilyin and E. G. Poznyak, Fundamentals of Mathematical Analysis, Mir Publishers, Moscow, 1982, 71-72.

ZOLTÁN TUZSON

REFORMED HIGH SCHOOL

535600 SZÉKELYUDVARHELY

ALEEA TEILOR $3 / 27$

ROMANIA

E-mail: tuzo60@gmail.com

(Received January, 2013) 